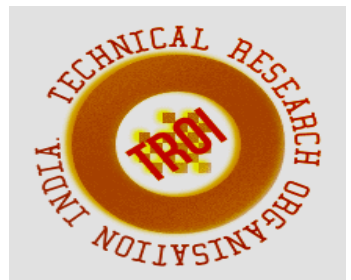

\title{
EVALUATION OF SOIL FERTILITY USING IMAGE PROCESSING
}

\author{
Manjushree $\mathrm{K}_{\text {Chavan }}{ }^{1}$, Anisha $\mathrm{V}^{2}$, Jeevika mary $\mathrm{A}^{3}$, Joyseelin priyanka $\mathrm{J}^{4}$, Monisha $\mathrm{K}^{5}$ \\ ${ }^{1}$ Assiatant Professor, ECE dept., Dr. T. Thimmaiah Institute of Technology, K.G.F \\ ${ }^{2,3,4,5}$ Students, ECE dept., Dr. T. Thimmaiah Institute of Technology,K.G.F \\ ${ }^{1}$ manjushree@drttit.edu.in, 3jeevikamary@gmail.com
}

\begin{abstract}
Agriculture is the backbone of India. Soil pH plays a very important role in finding the fertility status of the soil. The paper tells whether the soil is acidic, neutral or alkaline and its nutrients values like nitrogen, potassium, and calcium by using the database set in image processing. The obtained output is received through an SMS by using the GSM model (SIM800C).

Keywords: pH, color quantization, SVM Classifier, GSM
\end{abstract}

\section{Introduction}

India is known as a famous agricultural country. Most of the farmers do not perform soil testing because existing methods consumes time and money. Very few farmers rely on soil testing done by government labs which are not available near them. Soil testing is the way to know the quality of fertilizer to meet the necessity of the crop by taking advantage of nutrients already present in the soil. It will also help to know the solution for soil problems. The soil $\mathrm{pH}$ value plays an important role in soil analysis [1]. Soil $\mathrm{pH}$ tells us the acidity as well as basicity present in the soil because if the soil is very acidic then it will affect the growth of the plants. A pH value ranges from 1 to $14, \mathrm{pH}$ value below 7 is acidic whereas above 7 is alkaline. Soil $\mathrm{pH}$ is called as the main variable in the soil as it will control many chemical and biological processes that take place in the soil. The most suitable range for many plants is between 5.5 to 7.0. The importance of Soil testing plays an important role in the farmer's life. The acidity, neutrality or alkalinity of a soil is measured in terms of hydrogen ion. The main aim of finding the soil $\mathrm{pH}$ is that which crop is suitable for the soil.

\section{RELATED WORKS}

Amara denis M.K et al., have undertaken a detail soil survey with the aim of evaluating fertility states of the soil using nutrients index approach [2]. Based on the nutrients indices, soil reaction and $\mathrm{Fe}$ were observed as the most important soil fertility constraints that could affect sustainability crop production. Halimater sadiyah, Abduhali et al., proposed deep convolution neural network used to determine soil nutrients peculiarities. For example, the head . required in a plantation to optimize production[3]. They used maize plantation which was within average accuracy of $96 \%$.

Makara M Aziz et al., determine the $\mathrm{pH}$ of the soil by using a neural network based on soils colour [4]. The database suggested for this neural network stores the value of soil colour (RGB) and the $\mathrm{pH}$ value of each sample, this model showed good performance and it can be used to estimate the $\mathrm{pH}$ value.

Prof.Sangeetha et al., proposed the method for estimation, detection and comparison of soil nutrients analysis quantitatively by following the principle of chromatography techniques, at a different layer from the soil sample image processing of soil chromatogram, they found the soil nutrients and suggested the perfect fertilizer and suitable crop[5].

Umesh Kamble et al., proposed the number of fertilizers and $\mathrm{pH}$ of the soil that must be applied, soil samples were collected and their $\mathrm{pH}$ was tested in Govt testing lab[6]. On the basis of the RGB values, pixel properties, and digital correlation, the results of their $\mathrm{pH}$ values were matching with results of the testing lab. 
The $\mathrm{pH}$ value calculated by the software was slightly different than their tested value by $\mathrm{pH}$ meter

\section{METHODOLOGY}

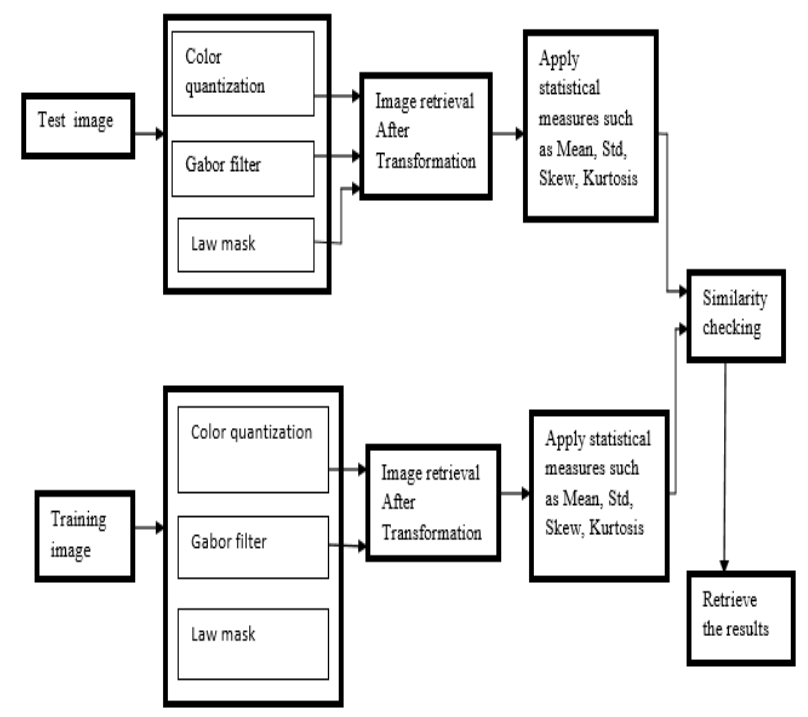

The methodology implemented is categorized into the following steps: first color quantization where it reduces the number of colors used in the image, the second step is applying Gabor filter used for texture analysis that it specifies the frequency content in the image in specific directions, third step is law mask technique for extraction of texture feature whose main approach is towards filtering the image with five types of mask namely level, edge, spot, ripple, and wave. Statistical measures such as mean, standard deviation, skew, and kurtosis are calculated and then it is followed by classifying the image retrieved by using SVM classifier and lastly the result is obtained.

\section{A. Color Quantization}

Color quantization reduces the number of color in the image and it is important for displaying a number of images on the device. Color quantization is viewed as a subset of the field of vector quantization. It is the problem of selecting $\mathrm{K}$ vectors in some $\mathrm{N}$-dimensional space. The process of color quantization is into four phases that are sampling the original image for color statistics mapping the color to its representative in the color map and quantizing the new image.

B. Gabor filter

Gabor filter is most interesting in the frequency domain, it is a Gaussian function shifted in frequency to the position at a distance from the origin in the orientation and is possible to obtain. Gabor wavelet that is most sensitive to narrowband frequency and orientation is possible to obtain an indicator for the given texture.

\section{Law mask}

Law mask is the texture energy measurement for the analysis of texture property of each pixel. Law mask identifies the properties to describe the texture such as uniformity, density, coarseness, roughness, regularity, linearity, directionality, direction, frequency, and phase. Law mask energy determines texture property by accessing average gray level, edges, spots, ripples and wave in texture[7].

\section{Statistical measures}

Statistical measures contain eight parameters such as Mean, Standard Deviation, Entropy, RMS, Variance, Smoothness, Kurtosis, Skewness.

Mean: It is used in geometry and analysis and they are used for spatial filtering and noise reduction.

Standard deviation: A standard deviation filter calculates the standard deviation and assigns this value to the center pixel in the output.

Entropy: It is a measure of randomness that can be used to characterize the texture of the input image.

RMS: It is a measure of the magnitude of a set of numbers it gives a sense for the typical size of the numbers.

Variance: Is expectation of the squared deviation of a random variable from its mean.

Smoothness: It is to create an approximating function that attempts to capture important patterns in the data leaving out the noise.

Kurtosis: it is used to describe the distribution with large kurtosis exhibit tail data exceeding the normal distribution.

Skewness: it measures how the pixel value is distributed[4].

SVM classifier: support vector machine is a supervised learning model with an associated learning algorithm that analyzes the data used for classification analysis.

SVM are essentially binary classifiers, they can be adapted to handle the multiple classification task SVM training algorithm builds a model that assigns new example to one category. It is a representation of the examples as points in space[8]. 


\section{E GSM MODULE}

The GSM

GPRS electronic

equipment has internal TCP/IP stack to alter to attach with net via GPRS.it is appropriate for SMS, voice similarly as information transfer application in M2M interface. The baud is configurable from 9600-115200 through AT command. The user gets the SMS that whether the soil is acidic, neutral or alkaline through the GSM module which is connected to the system.

\section{RESULT AND CONCLUSION}

The input image is quantized where it is converted to 16 color scales followed by kmeans clustering with $\mathrm{k}=16$ and statistical measures like Mean, Standard Deviation, Entropy, RMS, Variance, Smoothness, Kurtosis, Skewnessare found in order to analyze the features and texture of the input image. Gabor filter and law mask is applied where the statistical measure values are obtained. The same steps are followed for the set of 32 images (database) of training set. Using the SVM classifier the database is classified into 3 sets like acidic, neutral and alkaline where the output is categorized either in one of the set depending on the training image. The GSM model is used in order to send the SMS to the customer, to find what type of soil he has given for test and hence making the procedure for soil testing easier for the farmers.

Table 1: statistical Measures

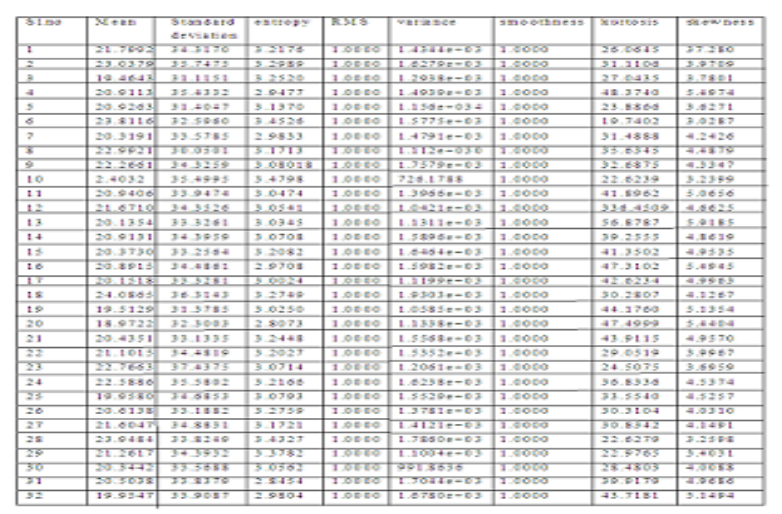

\section{REFERENCES}

[1] Umesh Kamble, pravin Shinge, "Testing of Agriculture Soil by Image Processing” International Journal of Scientific Research and Development Vol. 5, Issue 01, 2017

[2] Amara Denis M K, M. H. Uddin and Md. Serajul Islam,” Design and
Implementation of Microcontroller Based Digital Soil pH Meter", ULAB Journal of Science and Engineering Vol. 5, No. 1, November 2014 (Issn: 20794398)

[3] Halimater Sadiyah, Binod Kumar Vimal, RakeshKumar, RakeshKumar, Mukesh Kumar, "Determination of soil $\mathrm{pH}$ by using digital image processing technique," Journal of Applied and Natural Science, vol. 6, no. 1, pp. 14-18, 2014

[4] Makera M Aziz*. Dena Rafaa Ahmed, Banar Fareed Ibrahim,” Determine the Ph. of Soil by Using Neural Network Based on Soil's Color," International Journal of Advanced Research in Computer Science and Software Engineering Volume 6, Issue 11, November 2016 ISSN: 2277 128X.

[5] Professor Sangeetha, "Estimating surface soil moisture from soil color using image analysis," Vadose Zone Journal, vol. 4, p. 1119-1122, 2005

[6] Forrer. I, Papritz. A, Kasteel. R, Flühler. $\mathrm{H}$, Luca. D, "Quantifying dye tracers in soil profiles by image processing," European Journal of Soil Science, vol. 51, pp. 313-322, 2002.

[7] R. Shenbagavalli, K.Ramar, "Feature Extraction of Soil Images for Retrieval based on Statistics”IJCA Vol.88-No.14, 2014

[8] F. J. Sikora , P. Howe, D. Reid , D. Morgan \& E. Zimmer, "Adopting a Robotic pH Instrument for Soil and SoilBuffer pH Measurements in a Soil Test Laboratory," Communications in Soil Science and Plant Analysis, vol. 42, pp. 617-632, 2011.

[9] Vinay Kumar, Binod Kumar Vimal, RakeshKumar, RakeshKumar, Mukesh Kumar, "Determination of soil $\mathrm{pH}$ by using digital image processing technique," Journal of Applied and Natural Science, vol. 6, no. 1, pp. 14-18, 2014.

[10] M. A. A. Mashud, M. A. Masud, Md. Serajul Islam,” Design and development of microcontroller based digital $\mathrm{PH}$ meter”, ULAB Jounal of Science and Engineering, 2011, vol.4 pp31 